\title{
An improved empirical model for the effect of long-period ocean tides on polar motion
}

\author{
Richard S. Gross
}

Received: 20 May 2008 / Accepted: 30 September 2008 / Published online: 28 October 2008

(C) The Author(s) 2008. This article is published with open access at Springerlink.com

\begin{abstract}
Because the tide-raising potential is symmetric about the Earth's polar axis it can excite polar motion only by acting upon non-axisymmetric features of the Earth like the oceans. In fact, after removing atmospheric and non-tidal oceanic effects, polar motion excitation observations show a strong fortnightly tidal signal that is not completely explained by existing dynamical and empirical ocean tide models. So a new empirical model for the effect of the termensual ( $\mathrm{Mtm}$ and $\mathrm{mtm}$ ), fortnightly ( $\mathrm{M} f$ and $\mathrm{mf}$ ), and monthly $(\mathrm{Mm})$ tides on polar motion is derived here by fitting periodic terms at these tidal frequencies to polar motion excitation observations that span 2 January 1980 to 8 September 2006 and from which atmospheric and non-tidal oceanic effects have been removed. While this new empirical tide model can fully explain the observed fortnightly polar motion excitation signal during this time interval it would still be desirable to have a model for the effect of long-period ocean tides on polar motion that is determined from a dynamical ocean tide model and that is therefore independent of polar motion observations.
\end{abstract}

Keywords Earth rotation - Polar motion - Ocean tides . Oceanic angular momentum - Atmospheric angular Momentum

\section{Introduction}

The changing gravitational attraction of the Sun, Moon, and planets, besides causing the Earth to precess and nutate, also deforms the solid and fluid regions of the Earth, causing the Earth's inertia tensor to change and hence causing the Earth's

R. S. Gross (凶)

Jet Propulsion Laboratory, California Institute of Technology,

4800 Oak Grove Drive, Pasadena, CA 91109, USA

e-mail: Richard.Gross@jpl.nasa.gov rotation to change. Since the second-degree zonal tide raising potential is symmetric about the polar axis, the deformation of an axisymmetric Earth caused by this potential is also symmetric about the polar axis and therefore causes the Earth's rate of rotation and length-of-day to change but does not cause polar motion. Tidal changes in polar motion can only be excited by the action of the tide raising potential on non-axisymmetric features of the Earth like the oceans.

Ocean tides excite polar motion in the diurnal, semidiurnal, and long-period tidal bands. In fact, ocean tides in the diurnal and semidiurnal tidal bands are the principal cause of subdaily polar motion (Chao et al. 1996), and fortnightly ocean tides are the principal cause of intraseasonal polar motion once atmospheric and non-tidal oceanic effects are removed. To illustrate the importance of fortnightly ocean tides on polar motion, Fig. 1a shows a spectrum of polar motion excitation observations spanning 2 January 1993 to 10 September 2006, and Fig. 1b shows the spectrum after removing atmospheric and non-tidal oceanic effects. The most prominent peaks at intraseasonal frequencies in this residual spectrum are at the prograde and retrograde fortnightly tidal frequencies ( \pm 27 cycles per year).

Hydrodynamic ocean tide models have been used to predict the effects of long-period ocean tides on polar motion (Table 1) even before their effects were first observed by Chao (1994). For example, Dickman (1993) used his spherical harmonic tide model to predict the effect of 10 long-period ocean tidal constituents on polar motion. Figure 1c shows the spectrum of the result of removing the Dickman (1993) longperiod ocean tide model as tabulated by Gross et al. (1997) from the residual polar motion excitation observations whose spectrum is shown in Fig. 1b. As can be seen, the Dickman (1993) model reduces, but does not eliminate, the spectral peaks at the prograde and retrograde fortnightly tidal frequencies. As another example, Gross (1993) used the tabulated 


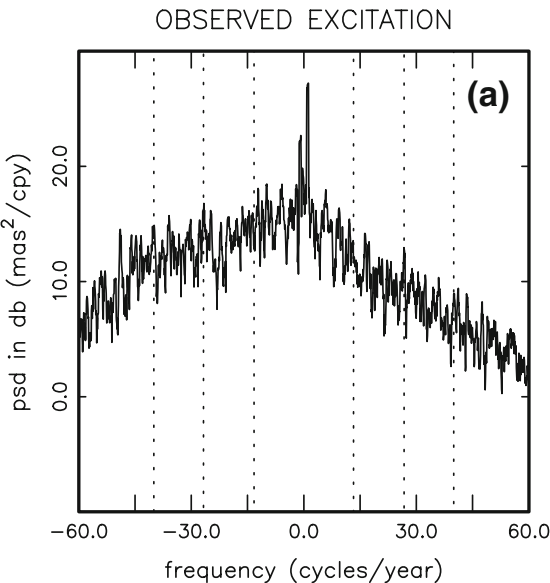

REMOVE DICKMAN DYNAMICAL MODEL

REMOVE WEIS DYNAMICAL MODEL
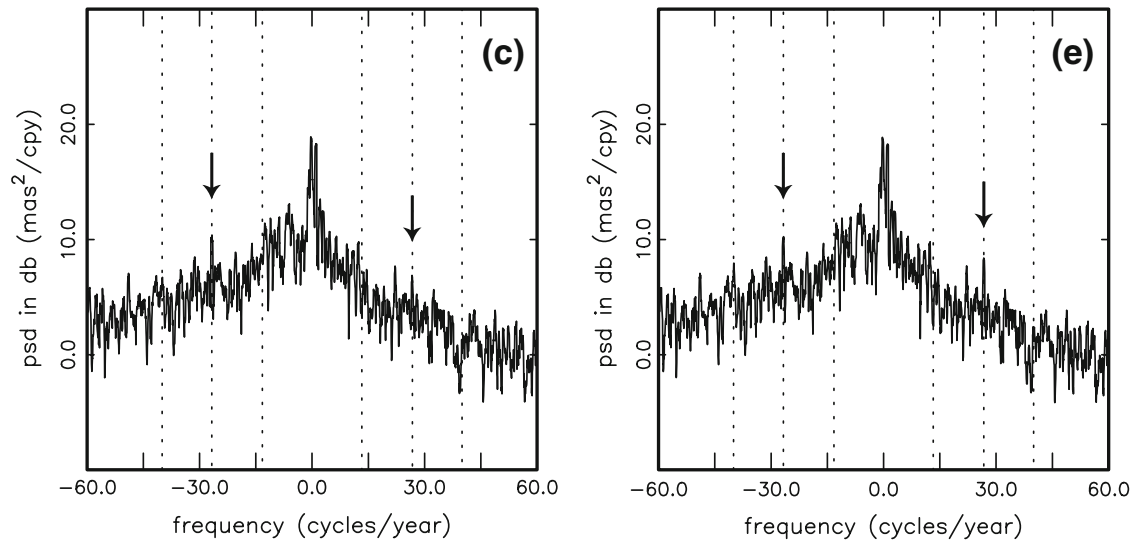

REMOVE AAM \& OAM

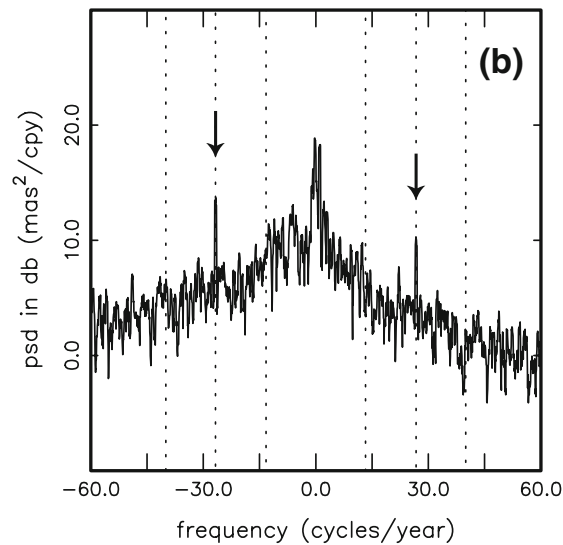

REMOVE G/S/B DYNAMICAL MODEL

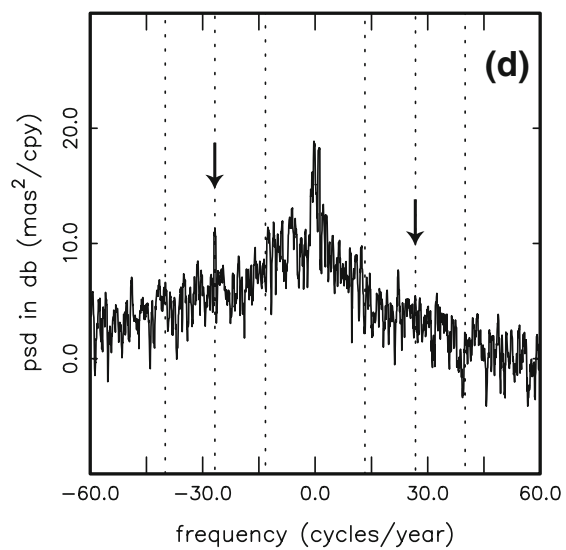

REMOVE 1997 EMPIRICAL MODEL

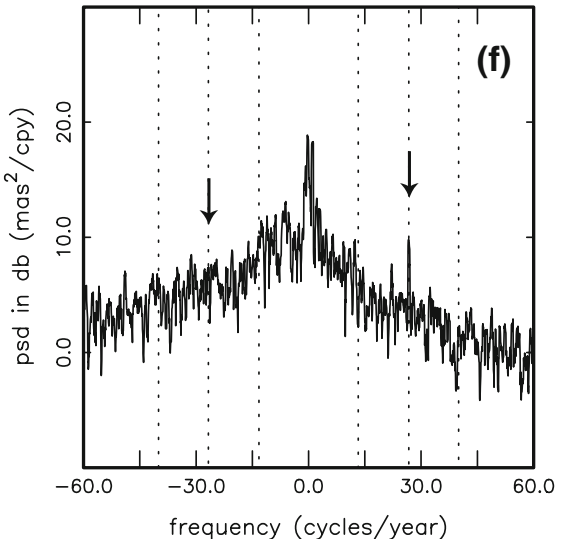

Fig. 1 Power spectral density (psd) estimates in decibels (db) computed by the multitaper method from time series of polar motion excitation functions $\chi(t)$ spanning 2 January 1993 to 10 September 2006 of the: a observed COMB2006 polar motion excitation functions; $\mathbf{b}$ residual polar motion excitation functions formed by removing atmospheric and non-tidal oceanic effects from the observed functions; c residual excitation functions formed by additionally removing the Dickman (1993) tide model as reported by Gross et al. (1997); d residual excitation functions formed by additionally removing the Seiler (1991) tide model as reported by Gross (1993); e residual excitation functions formed by additionally removing the Weis (2006) tide model; and f residual excitation functions formed by additionally removing the empirical model of Gross et al. (1997). Vertical dashed lines indicate the prograde and retrograde frequencies of the termensual ( \pm 40 cpy), fortnightly ( \pm 27 cpy) and monthly $( \pm 13 \mathrm{cpy})$ tidal terms. The prograde and retrograde fortnightly tidal terms are also indicated by arrows. The retrograde component of polar motion excitation is represented by negative frequencies, the prograde component by positive frequencies ocean tidal angular momenta results of Seiler (1991), an extension of the results of Brosche (1982), to predict the effect of the fortnightly, monthly, and semiannual ocean tides on polar motion. Figure 1d shows the spectrum of the result of removing the Gross/Seiler/Brosche long-period ocean tide model as tabulated by Gross et al. (1997) from the residual polar motion excitation observations whose spectrum is shown in Fig. 1b. While this model does an excellent job of removing the observed power at the prograde fortnightly tidal frequency, it does not completely remove the power at the retrograde fortnightly tidal frequency. As a final example, Weis (2006) recently developed a high-resolution global ocean tide model and used this model to predict the effect of 12 long-period ocean tidal constituents on polar motion. Figure 1e shows the spectrum of the result of removing the Weis (2006) long-period ocean tide model from the residual polar motion excitation observations whose spectrum is shown in Fig. 1b. As can be seen, the Weis (2006) model is also unable to completely explain the observed power at either the prograde or retrograde fortnightly tidal frequencies.

The hydrodynamic ocean tide models discussed above are not constrained by measurements, either from tide gauges or from satellite altimeters. While such measurements have been successfully used to constrain models of the effect of the semidiurnal and diurnal ocean tides on polar motion (Chao et al. 1996; Chao and Ray 1997), they have not yet been successfully used to constrain models of the effect of the 
Table 1 Ocean tidal variations in polar motion and polar motion excitation

\begin{tabular}{|c|c|c|c|c|c|c|c|c|c|c|c|c|c|c|}
\hline \multirow[t]{3}{*}{ Tide model } & \multicolumn{5}{|c|}{ Argument } & \multirow{3}{*}{$\begin{array}{l}\text { Period } \\
\text { (days) }\end{array}$} & \multicolumn{4}{|c|}{ Polar motion } & \multicolumn{4}{|c|}{ Polar motion excitation } \\
\hline & \multirow[t]{2}{*}{$l$} & \multirow[t]{2}{*}{$l^{\prime}$} & \multirow[t]{2}{*}{$F$} & \multirow[t]{2}{*}{$D$} & \multirow[t]{2}{*}{$\Omega$} & & \multicolumn{2}{|c|}{ Prograde } & \multicolumn{2}{|c|}{ Retrograde } & \multicolumn{2}{|c|}{ Prograde } & \multicolumn{2}{|c|}{ Retrograde } \\
\hline & & & & & & & $\begin{array}{l}\text { Amp } \\
\text { ( } \mu \text { as) }\end{array}$ & $\begin{array}{l}\text { Phase } \\
\text { (degrees) }\end{array}$ & $\begin{array}{l}\text { Amp } \\
\text { ( } \mu \text { as) }\end{array}$ & $\begin{array}{l}\text { Phase } \\
\text { (degrees) }\end{array}$ & $\begin{array}{l}\text { Amp } \\
\text { ( } \mu \text { as) }\end{array}$ & $\begin{array}{l}\text { Phase } \\
\text { (degrees) }\end{array}$ & $\begin{array}{l}\text { Amp } \\
\text { ( } \mu \text { as) }\end{array}$ & $\begin{array}{l}\text { Phase } \\
\text { (degrees) }\end{array}$ \\
\hline \multicolumn{15}{|l|}{$m t m$} \\
\hline Dickman & 1 & 0 & 2 & 0 & 1 & 9.12 & 2.81 & -107.10 & 4.40 & 15.21 & 130.38 & 72.73 & 213.45 & 15.05 \\
\hline Weis & 1 & 0 & 2 & 0 & 1 & 9.12 & 5.34 & 159.00 & 3.76 & -137.00 & 248.17 & -21.16 & 182.26 & -137.16 \\
\hline 1997 Emp & 1 & 0 & 2 & 0 & 1 & 9.12 & 14.65 & 174.32 & 12.47 & 140.84 & 680.82 & -5.84 & 604.66 & 140.69 \\
\hline This paper & 1 & 0 & 2 & 0 & 1 & 9.12 & 3.88 & -128.58 & 1.65 & -26.83 & 180.15 & 51.26 & 80.18 & -26.98 \\
\hline \multicolumn{15}{|l|}{ Mtm } \\
\hline Dickman & 1 & 0 & 2 & 0 & 2 & 9.13 & 6.79 & -106.95 & 10.69 & 15.32 & 315.24 & 72.89 & 517.67 & 15.17 \\
\hline Weis & 1 & 0 & 2 & 0 & 2 & 9.13 & 18.47 & -144.00 & 11.07 & 103.00 & 857.20 & 35.84 & 535.91 & 102.84 \\
\hline 1997 Emp & 1 & 0 & 2 & 0 & 2 & 9.13 & 4.73 & -161.12 & 6.84 & -36.41 & 219.41 & 18.71 & 330.95 & -36.57 \\
\hline This paper & 1 & 0 & 2 & 0 & 2 & 9.13 & 9.36 & -128.58 & 4.00 & -26.83 & 434.55 & 51.26 & 193.41 & -26.98 \\
\hline \multicolumn{15}{|l|}{$m f$} \\
\hline Dickman & 0 & 0 & 2 & 0 & 1 & 13.63 & 16.95 & -79.91 & 21.77 & 8.01 & 521.36 & 99.93 & 713.09 & 7.85 \\
\hline $\mathrm{G} / \mathrm{S} / \mathrm{B}$ & 0 & 0 & 2 & 0 & 1 & 13.63 & 23.30 & -125.00 & 18.00 & 72.00 & 716.71 & 54.83 & 589.68 & 71.84 \\
\hline Weis & 0 & 0 & 2 & 0 & 1 & 13.63 & 28.85 & -154.00 & 23.39 & 83.00 & 887.43 & 25.83 & 766.26 & 82.84 \\
\hline 1997 Emp & 0 & 0 & 2 & 0 & 1 & 13.63 & 44.42 & -149.39 & 41.65 & 69.17 & 1366.47 & 30.44 & 1364.30 & 69.02 \\
\hline This paper & 0 & 0 & 2 & 0 & 1 & 13.63 & 28.62 & -105.95 & 36.81 & 39.42 & 880.22 & 73.88 & 1205.93 & 39.27 \\
\hline \multicolumn{15}{|l|}{$M f$} \\
\hline Dickman & 0 & 0 & 2 & 0 & 2 & 13.66 & 40.96 & -79.76 & 52.49 & 7.97 & 1257.22 & 100.08 & 1716.11 & 7.82 \\
\hline $\mathrm{G} / \mathrm{S} / \mathrm{B}$ & 0 & 0 & 2 & 0 & 2 & 13.66 & 56.10 & -125.00 & 44.00 & 72.00 & 1722.07 & 54.83 & 1438.64 & 71.84 \\
\hline Weis & 0 & 0 & 2 & 0 & 2 & 13.66 & 100.07 & -132.00 & 95.03 & 71.00 & 3071.79 & 47.83 & 3107.14 & 70.84 \\
\hline 1997 Emp & 0 & 0 & 2 & 0 & 2 & 13.66 & 55.16 & -64.07 & 79.01 & 31.48 & 1693.09 & 115.76 & 2583.21 & 31.32 \\
\hline This paper & 0 & 0 & 2 & 0 & 2 & 13.66 & 69.18 & -105.95 & 88.97 & 39.42 & 2123.44 & 73.88 & 2909.16 & 39.27 \\
\hline \multicolumn{15}{|l|}{$M m$} \\
\hline Dickman & 1 & 0 & 0 & 0 & 0 & 27.56 & 31.61 & -43.97 & 16.98 & -6.35 & 465.13 & 135.86 & 283.75 & -6.50 \\
\hline $\mathrm{G} / \mathrm{S} / \mathrm{B}$ & 1 & 0 & 0 & 0 & 0 & 27.56 & 53.00 & -106.00 & 55.00 & 28.00 & 779.85 & 73.83 & 919.28 & 27.85 \\
\hline Weis & 1 & 0 & 0 & 0 & 0 & 27.56 & 46.29 & -77.00 & 50.17 & 9.00 & 681.12 & 102.83 & 838.55 & 8.85 \\
\hline 1997 Emp & 1 & 0 & 0 & 0 & 0 & 27.56 & 43.97 & -85.01 & 58.62 & -55.19 & 646.96 & 94.82 & 979.84 & -55.34 \\
\hline This paper & 1 & 0 & 0 & 0 & 0 & 27.56 & 38.56 & -111.41 & 83.24 & 34.77 & 567.37 & 68.42 & 1391.36 & 34.62 \\
\hline
\end{tabular}

$l, l, F, D$, and $\Omega$ are the Delaunay arguments of the tidal constituent, expressions for which are given in Simon et al. (1994). The period, given in solar days, is the approximate period of the term as viewed in the terrestrial reference frame. The amplitude (amp) and phase of the prograde and retrograde components of polar motion excitation are defined by Eq. (3). The amplitude and phase of the prograde and retrograde components of polar motion are similarly defined but with $\mathbf{p}(t)=p_{x}(t)-i p_{y}(t)$. The units of the amplitudes are microarcseconds ( $\mu$ as). The tabulated entries for the tide model labeled "Dickman" are from Dickman (1993) as reported by Gross et al. (1997); the entries labeled "G/S/B" are from Seiler (1991) as reported by Gross (1993); the entries labeled "Weis" are from Weis (2006); the entries labeled "1997 Emp" are from the empirical model of Gross et al. (1997); and the entries labeled "This paper" are from the preferred empirical tide model determined in this paper

long-period ocean tides. Desai and Wahr (1995) estimated a long-period ocean tide model from Topex/Poseidon altimetric sea surface height measurements and Gross et al. (1997) used this model to predict the effect of the fortnightly and monthly ocean tides on polar motion excitation. But they noticed that as additional Topex/Poseidon measurements were incorporated into the ocean tide model the predicted effect changed. That is, the predicted effect had not converged, thus raising doubts about the accuracy of the predictions.
Besides hydrodynamic ocean tide models, empirical models based upon fitting to the observations periodic terms at the tidal frequencies have also been developed (Gross et al. 1996; Gross et al. 1997). Figure 1f shows the spectrum of the result of removing the Gross et al. (1997) empirical longperiod tide model from the residual polar motion excitation observations whose spectrum is shown in Fig. 1b. While this empirical model does an excellent job of removing the observed power at the retrograde fortnightly tidal frequency, it does 
not completely remove the power at the prograde fortnightly tidal frequency.

Gross et al. (1997) determined their empirical model by least-squares fitting periodic terms at the main long-period tidal frequencies to polar motion excitation observations from which atmospheric effects were removed. The tidal constituents fit were the two principal termensual terms (Mtm and $m t m$ ), the two principal fortnightly terms ( $M f$ and $m f$ ), and the principal monthly term $(\mathrm{Mm})$. Since the frequencies of the $M f$ and $m f$ tidal terms differ by only $1 / 18.6$ cycles per year (cpy), the oceans and hence the Earth's rotation should have the same relative response to the tide raising potential at these frequencies. That is, the $M f$ and $m f$ polar motion excitation tidal terms of the empirical model of Gross et al. (1997) should have the same phase and an amplitude ratio that is the same as that of the tide raising potential at these frequencies. But as discussed by Gross et al. (1997) and as can be seen from the entries for that model in Table 1 they do not, and neither do their results for the Mtm and $\mathrm{mtm}$ polar motion excitation tidal terms that also differ by only 1/18.6cpy in frequency. This inconsistency of the Gross et al. (1997) empirical tide model has always been worrisome and may be the root cause of its inability to fully account for the fortnightly tidal power that is observed during 2 January 1993 to 10 September 2006 (Fig. 1f).

Here, an improved empirical model for the effect of the termensual, fortnightly, and monthly ocean tides on polar motion excitation is obtained by least-squares fitting periodic terms at these tidal frequencies to polar motion excitation observations that span 2 January 1980 to 8 September 2006 and from which both atmospheric and non-tidal oceanic effects have been removed. During this fit the periodic terms at the $M f$ and $m f$ tidal frequencies are constrained to have the same phase and an amplitude ratio that is the same as that of the tide raising potential at these frequencies; the same constraints are applied to the periodic terms at the $\mathrm{Mtm}$ and $m t m$ tidal frequencies.

\section{Data sets}

The observed polar motion excitation series used in this study is from the COMB2006 combined Earth orientation series (Gross 2007). COMB2006 is a combination of Earth orientation measurements taken by the techniques of optical astrometry, lunar and satellite laser ranging, very long baseline interferometry, and the global positioning system. The Kalman filter used to combine the polar motion measurements also estimates their time rate-of-change, thereby allowing the polar motion excitation functions to be determined. The COMB2006 polar motion excitation series spans 20 January 1962 to 10 February 2007 at daily intervals. Figure 1a shows the spectrum of that portion of the COMB2006 polar motion excitation series spanning 2 January 1993 to 10 September 2006.

The effects of atmospheric winds and surface pressure variations were removed from the COMB2006 polar motion excitation series using effective atmospheric angular momentum (AAM) functions that were computed from products of the National Centers for Environmental Prediction/National Center for Atmospheric Research (NCEP/NCAR) reanalysis project (Kalnay et al. 1996). The pressure term used here assumes that the oceans respond as an inverted barometer to the imposed atmospheric surface pressure variations. The NCEP/NCAR reanalysis AAM series, which was obtained from the International Earth Rotation and Reference Systems Service (IERS) Special Bureau for the Atmosphere (Salstein 2003) and which was computed by them following the procedures of Zhou et al. (2006) using the formulation of Eubanks (1993), is given at 6-hour intervals since 1 January 1948. Daily averages of the 6-hour values were formed by summing 5 consecutive values using weights of $1 / 8,1 / 4,1 / 4$, $1 / 4,1 / 8$

The effects of oceanic currents and the resulting bottom pressure variations were removed from the polar motion excitation observations using effective oceanic angular momentum (OAM) functions computed from the products of two different unconstrained oceanic general circulation models (OGCMs). Both models were run at the Jet Propulsion Laboratory (JPL) as part of their participation in the Estimating the Circulation and Climate of the Oceans (ECCO) consortium (Stammer et al. 2002). The first model, designated kf066a2, is the unconstrained version of model kf066b. Both kf066a2 and kf066b have the same model configuration and forcing as kf049f, the older constrained ECCO/JPL model that was replaced by the newer kf066b model. That is, both kf066a 2 and kf066b are based on the MIT oceanic general circulation model (Marshall et al. 1997a,b), have realistic boundaries and bottom topography, have 46 levels ranging in thickness from $10 \mathrm{~m}$ at the surface to $400 \mathrm{~m}$ at depth using height as the vertical coordinate, and span the globe between $80^{\circ} \mathrm{S}$ to $80^{\circ} \mathrm{N}$ latitude with a latitudinal grid-spacing ranging between $0.3^{\circ}$ at the equator to $1^{\circ}$ at the poles and a longitudinal grid-spacing of 1 degree. During the 10-year spin-up period the models were forced with climatological fields from the Comprehensive Ocean-Atmosphere Data Set (COADS). Subsequently, the models were forced with twice daily wind stress and daily surface heat flux and evaporation-precipitation fields from the NCEP/NCAR reanalysis project.

Atmospheric surface pressure fields were not used to force the models. At periods greater than a few days, and almost certainly at the termensual (9-day) to monthly periods of interest here, the predominant response of the oceans to surface pressure forcing is a simple static response like that of an inverted barometer (Wunsch and Stammer 1997). The static response of the oceans to changes in atmospheric surface 
pressure was taken into account here when atmospheric effects were removed from the polar motion excitation series.

Model kf066b assimilates altimetric sea surface height measurements whereas model kf066a2 does not assimilate any measurements. Even though one might think that kf066b is the more realistic model of the general circulation of the oceans because it is constrained by data, it was not used here to remove the effects of non-tidal oceanic current and bottom pressure variations from the polar motion excitation observations because, like kf049f, it contains tidal signals. A tide model was used to remove tidal signals from the altimetric sea surface height measurements before they were assimilated into kf049f and kf066b. Unfortunately, before July 2005 an equilibrium model was used to remove the long-period tidal signal (Fukumori, personal communication, 2007), thereby allowing the non-equilibrium component of the longperiod tides to be assimilated into kf049f and kf066b. This introduced a tidal signal into the bottom pressure fields of these models and hence into the oceanic angular momentum computed from the bottom pressure fields. So, the unconstrained model kf066a2, which does not contain any tidal signals, was used here to remove non-tidal oceanic current and bottom pressure effects from the polar motion excitation observations. The OAM series computed here from the oceanic current and bottom pressure fields of model kf066a 2 spans 2 January 1993 to 10 September 2006 at daily intervals.

In order to test the sensitivity of the empirical polar motion excitation tide model determined in this paper to the ocean model that is used to remove non-tidal oceanic effects from the polar motion excitation observations, another unconstrained ECCO/JPL ocean model was examined. This model, designated c20010701, is the same as that studied by Gross et al. (2003). It has the same model configuration and forcing as kf066a 2 except that its latitudinal domain is slightly smaller, extending from $74^{\circ} \mathrm{S}$ to $74^{\circ} \mathrm{N}$ latitude. The OAM series computed from the oceanic current and bottom pressure fields of this model, which was obtained from the IERS Special Bureau for the Oceans (Gross 2003), spans 2 January 1980 to 31 March 2002 at daily intervals.

Both kf066a2 and c20010701 are based on the MIT oceanic general circulation model that, because it is formulated using the Boussinesq approximation (Marshall et al. 1997a,b), conserves volume rather than mass. Artificial mass variations can be introduced into Boussinesq models through the applied surface heat and salt fluxes. For example, the changing applied heat flux will change the density, which, since volume is conserved, will artificially change the mass of the modeled oceans. If left uncorrected, this artificial mass change will cause artificial changes to the ocean-bottom pressure and hence to the angular momentum computed from the bottom pressure variations. The artificial mass variations caused by the use of the Boussinesq approximation have been removed from the $\mathrm{kf066 \textrm {a } 2}$ and $\mathrm{c} 20010701$ models by separately computing the effect on the bottom pressure of a spatially uniform layer added to each model's sea surface that has just the right fluctuating thickness to impose mass conservation on that model (Greatbatch 1994).

A third OAM series was formed by splicing together the Boussinesq-corrected c20010701 and kf066a2 series in order to generate a series that spans the longest possible time interval. This spliced series was created by concatenating that segment of the c20010701 series spanning 2 January 1980 to 1 January 1993 with the entire kf066a2 series after correcting the entire kf066a 2 series to have the same trend as that exhibited by c20010701 during their common, overlapping time interval. The resulting spliced OAM series spans 2 January 1980 to 10 September 2006 at daily intervals.

Figure 1b shows the spectrum of the COMB2006 polar motion excitation observations from which atmospheric and non-tidal oceanic effects have been removed using the NCEP/ NCAR reanalysis AAM and the kf066a2 OAM series, respectively. The two largest peaks in this spectrum on intraseasonal time scales, indicated by the arrows, are at the prograde and retrograde fortnightly tidal frequencies ( \pm 27 cpy). Because the power of the AAM and OAM series is broadband it includes power in the fortnightly tidal band. So when the AAM and OAM series are removed from the observations the power in the fortnightly tidal band is reduced along with the background power, thereby causing the fortnightly tidal peaks to be slightly smaller in Fig. 1b than they are in Fig. 1a. While no spectral peaks are evident at the termensual ( \pm 40 cpy) or monthly ( \pm 13 cpy) tidal frequencies, periodic terms at these frequencies will nevertheless be included in the least-squares fit for the empirical polar motion excitation tide model.

\section{Polar motion excitation tide model}

\subsection{Method of determination}

An empirical model for the effect of the termensual (Mtm and $\mathrm{mtm}$ ), fortnightly ( $M f$ and $m f$ ), and monthly $(\mathrm{Mm}$ ) ocean tides on polar motion is obtained here by least-squares fitting periodic terms at these tidal frequencies to residual polar motion excitation observations, that is, to observations from which atmospheric and non-tidal oceanic effects have been removed. A linear trend and periodic terms at the semiannual $(S s a)$ and annual $(S a)$ tidal frequencies were also included in this fit. But the results for the $S s a$ and $S a$ tidal terms will not be discussed here because they also include non-tidal effects caused by, for example, seasonal changes in water stored on land.

The model fit to the $x$-component $\chi_{x}$ of the residual polar motion excitation observations is: 
$\chi_{x}(t)=a+b\left(t-t_{o}\right)+\sum_{i=1}^{N} A_{i} \cos \left[\alpha_{i}(t)-\phi_{i}\right]$

where $a$ is the bias term, $b$ is the coefficient of the linear rate term, $t_{o}$ is the reference epoch of the rate term, $A_{i}$ is the amplitude of the $i$ th tidal term, $\phi_{i}$ is the phase of the $i$ th tidal term, $\alpha_{i}$ is the argument of the $i$ th tidal term, and the sum extends over the number $N$ of tidal terms being fit to the residual observations. The same model is, of course, separately fit to the $y$-component.

Constraints are applied to tidal terms that have nearly the same frequency and that should therefore have the same relative response to the tide raising potential. This is done by setting the phases of the tidal terms to be equal to each other $\left(\phi_{1}=\phi_{2}\right)$ and by setting the ratio of the amplitudes of the tidal terms to be equal to the ratio of the amplitudes of the tide raising potential at the frequencies of those terms $\left(A_{1} / A_{2}=f\right)$. The model fit to the residual observations then becomes:

$$
\begin{aligned}
\chi_{x}(t)= & a+b\left(t-t_{o}\right)+\sum_{i=3}^{N} A_{i} \cos \left[\alpha_{i}(t)-\phi_{i}\right] \\
& +A_{2} \sin \phi_{2}\left(\sin \alpha_{2}+f \sin \alpha_{1}\right) \\
& +A_{2} \cos \phi_{2}\left(\cos \alpha_{2}+f \cos \alpha_{1}\right)
\end{aligned}
$$

where the sum now extends over just those terms that are not being constrained. The parameters of the constrained tidal terms that are determined by fitting this model to the residual observations are the amplitude $A_{2}$ and phase $\phi_{2}$ of the second constrained tidal term. The amplitude $A_{1}$ and phase $\phi_{1}$ of the first constrained tidal term can be obtained by setting $\phi_{1}=\phi_{2}$ and $A_{1}=f A_{2}$. This constraint is applied to the $M f$ and $m f$ tidal terms and, separately, to the Mtm and $\mathrm{mtm}$ tidal terms. According to Cartwright and Edden (1973), the $m f / M f$ ratio $f$ of the amplitudes of the tide raising potential is 0.4145 , and the $\mathrm{mtm} / \mathrm{Mtm}$ ratio is 0.4146 .

\subsection{Trial models}

A number of different trial models were obtained by using both weighted and unweighted least-squares fits, by using constrained and unconstrained fits, by fitting to observations spanning different time intervals, and by removing non-tidal oceanic effects using the c20010701 oceanic general circulation model, the kf066a2 model, and a model formed by splicing together these two models. Table 2 gives the coefficients of these trial models in terms of the amplitude $A$ and phase $\phi$ of the prograde (subscript $p$ ) and retrograde (subscript $r$ ) components of polar motion excitation defined by:

$$
\begin{aligned}
\chi(t) & =\chi_{x}(t)+i \chi_{y}(t) \\
& =A_{p} e^{i \phi_{p}} e^{i \alpha(t)}+A_{r} e^{i \phi_{r}} e^{-i \alpha(t)}
\end{aligned}
$$

where $\alpha(t)$ is the tidal argument, the expansion of which is given in Table 1.

The results of the trial models obtained by unweighted least-squares fits are not shown in Table 2. Because the accuracy of the polar motion excitation observations varies with time it is more appropriate to use weighted least-squares fits to determine the empirical tide model. For the weighted fits the formal uncertainties of the COMB2006 polar motion excitation series were used as weights. Because the AAM and OAM series used here to remove atmospheric and nontidal oceanic effects from the observations do not include uncertainties, the only uncertainties available to assign to the residual polar motion excitation series were the formal uncertainties of the COMB2006 observed polar motion excitation series.

The differences in the results for $M f$ given in Table 2 are reasonably close to each other when different OGCMs are used to remove the non-tidal oceanic effects from the observations. For example, depending on the OGCM, the constrained solutions for the prograde (amplitudes, phases) differ by at most $\left(112 \mu \mathrm{as}, 19^{\circ}\right)$ and the constrained solutions for the retrograde (amplitudes, phases) differ by at most ( $309 \mu$ as, $11^{\circ}$ ). These differences in value are within the 3 -sigma formal uncertainties of their determination. Similar differences are found between the unconstrained solutions when different OGCMs are used to remove the non-tidal oceanic effects.

The results for $M m$ given in Table 2 also show little dependence on the particular OGCM that is used to remove the non-tidal oceanic effects from the observations. For example, depending on the OGCM, the constrained solutions for the prograde (amplitudes, phases) differ by at most $\left(69 \mu\right.$ as, $\left.15^{\circ}\right)$ and the constrained solutions for the retrograde (amplitudes, phases) differ by at most $\left(236 \mu\right.$ as, $\left.13^{\circ}\right)$. These differences in value are within the 2-sigma formal uncertainties of their determination.

The effect of the Mtm tide on polar motion excitation is not as well determined as the effects of the $M f$ and $M m$ tides because its amplitude, and hence its signal-to-noise ratio, is much smaller than that of the $M f$ and $M m$ tides, particularly for the retrograde component. The solutions therefore show greater sensitivity to the particular OGCM that is used to remove non-tidal oceanic effects from the polar motion excitation observations. For example, depending on the OGCM, the constrained solutions for the retrograde phases differ by as much as $58^{\circ}$. While this is within the 2-sigma uncertainty of the phase determination, it is more than five times larger than the $11^{\circ}$ difference between the constrained solutions for the retrograde $M f$ phases.

Not surprisingly, the results for $\mathrm{Mm}$ shown in Table 2 do not depend on whether or not constraints are applied to the amplitudes and phases of $M f$ and $m f$ (and Mtm and $m t m$ ) during the weighted least-squares fit. Constraining the solution has the largest impact on the terms being constrained. 
Table 2 Trial empirical polar motion excitation tide models

\begin{tabular}{|c|c|c|c|c|c|c|}
\hline \multirow{2}{*}{$\begin{array}{l}\text { Non-Tidal } \\
\text { OGCM }\end{array}$} & \multirow[t]{2}{*}{ Constraint } & \multirow{2}{*}{$\begin{array}{l}\text { Data } \\
\text { Span }\end{array}$} & \multicolumn{2}{|l|}{ Prograde excitation } & \multicolumn{2}{|c|}{ Retrograde excitation } \\
\hline & & & Amplitude, $\mu$ as & Phase, deg & Amplitude, $\mu$ as & Phase, deg \\
\hline \multicolumn{7}{|l|}{$m t m$} \\
\hline c20010701 & Unconstrained & 1980-2002 & $453.70 \pm 152.97$ & $-28.84 \pm 19.32$ & $365.62 \pm 153.02$ & $111.91 \pm 23.97$ \\
\hline kf066a2 & Unconstrained & 1993-2006 & $476.40 \pm 141.64$ & $15.55 \pm 17.04$ & $140.34 \pm 141.69$ & $85.94 \pm 57.82$ \\
\hline spliced & Unconstrained & 1980-2006 & $429.01 \pm 122.85$ & $2.69 \pm 16.41$ & $290.70 \pm 122.90$ & $129.43 \pm 24.21$ \\
\hline c20010701 & Constrained & 1980-2002 & 125.94 & 55.12 & 115.19 & 2.67 \\
\hline kf066a2 & Constrained & 1993-2006 & 180.56 & 70.35 & 168.72 & -54.85 \\
\hline spliced & Constrained & 1980-2006 & 180.15 & 51.26 & 80.18 & -26.98 \\
\hline \multicolumn{7}{|l|}{ Mtm } \\
\hline c20010701 & Unconstrained & 1980-2002 & $408.21 \pm 152.99$ & $59.84 \pm 21.47$ & $367.62 \pm 153.01$ & $2.62 \pm 23.84$ \\
\hline kf066a2 & Unconstrained & 1993-2006 & $601.65 \pm 141.65$ & $85.11 \pm 13.49$ & $536.12 \pm 141.66$ & $-44.90 \pm 15.14$ \\
\hline spliced & Unconstrained & 1980-2006 & $530.54 \pm 122.88$ & $69.20 \pm 13.27$ & $377.87 \pm 122.89$ & $-16.64 \pm 18.63$ \\
\hline c20010701 & Constrained & 1980-2002 & $303.77 \pm 149.69$ & $55.12 \pm 28.24$ & $277.86 \pm 149.71$ & $2.67 \pm 30.86$ \\
\hline kf066a2 & Constrained & 1993-2006 & $435.52 \pm 123.20$ & $70.35 \pm 16.21$ & $406.96 \pm 123.20$ & $-54.85 \pm 17.35$ \\
\hline spliced & constrained & 1980-2006 & $434.55 \pm 106.92$ & $51.26 \pm 14.10$ & $193.41 \pm 106.93$ & $-26.98 \pm 31.68$ \\
\hline \multicolumn{7}{|l|}{$m f$} \\
\hline c20010701 & Unconstrained & 1980-2002 & $1196.46 \pm 153.01$ & $97.34 \pm 7.33$ & $1203.18 \pm 153.02$ & $37.46 \pm 7.28$ \\
\hline kf066a2 & Unconstrained & 1993-2006 & $960.69 \pm 141.64$ & $71.61 \pm 8.45$ & $1151.33 \pm 141.70$ & $56.47 \pm 7.05$ \\
\hline spliced & Unconstrained & 1980-2006 & $1101.19 \pm 122.87$ & $71.02 \pm 6.39$ & $1210.89 \pm 122.90$ & $55.14 \pm 5.81$ \\
\hline c20010701 & Constrained & 1980-2002 & 902.68 & 89.06 & 1333.92 & 28.49 \\
\hline kf066a2 & Constrained & 1993-2006 & 856.24 & 69.86 & 1219.68 & 39.51 \\
\hline spliced & Constrained & 1980-2006 & 880.22 & 73.88 & 1205.93 & 39.27 \\
\hline \multicolumn{7}{|l|}{$M f$} \\
\hline c20010701 & Unconstrained & 1980-2002 & $2114.17 \pm 152.98$ & $89.91 \pm 4.15$ & $3274.54 \pm 153.00$ & $28.68 \pm 2.68$ \\
\hline kf066a2 & Unconstrained & 1993-2006 & $2024.07 \pm 141.66$ & $70.89 \pm 4.01$ & $3125.64 \pm 141.65$ & $38.68 \pm 2.60$ \\
\hline spliced & Unconstrained & 1980-2006 & $2058.38 \pm 122.87$ & $76.54 \pm 3.42$ & $3041.01 \pm 122.88$ & $37.05 \pm 2.32$ \\
\hline c20010701 & Constrained & 1980-2002 & $2177.61 \pm 149.69$ & $89.06 \pm 3.94$ & $3217.93 \pm 149.70$ & $28.49 \pm 2.67$ \\
\hline kf066a2 & Constrained & 1993-2006 & $2065.57 \pm 123.21$ & $69.86 \pm 3.42$ & $2942.33 \pm 123.21$ & $39.51 \pm 2.40$ \\
\hline spliced & Constrained & 1980-2006 & $2123.44 \pm 106.92$ & $73.88 \pm 2.89$ & $2909.16 \pm 106.93$ & $39.27 \pm 2.11$ \\
\hline \multicolumn{7}{|l|}{$M m$} \\
\hline c20010701 & Unconstrained & 1980-2002 & $565.40 \pm 143.79$ & $54.77 \pm 14.55$ & $1284.96 \pm 143.56$ & $47.67 \pm 6.41$ \\
\hline kf066a2 & Unconstrained & 1993-2006 & $635.13 \pm 130.54$ & $70.08 \pm 11.77$ & $1521.18 \pm 130.40$ & $36.01 \pm 4.92$ \\
\hline spliced & Unconstrained & 1980-2006 & $566.97 \pm 117.31$ & $68.47 \pm 11.85$ & $1392.28 \pm 117.21$ & $34.60 \pm 4.83$ \\
\hline c20010701 & Constrained & 1980-2002 & $566.34 \pm 143.78$ & $54.74 \pm 14.53$ & $1284.50 \pm 143.56$ & $47.65 \pm 6.41$ \\
\hline kf066a2 & Constrained & 1993-2006 & $635.39 \pm 130.54$ & $70.06 \pm 11.76$ & $1520.19 \pm 130.40$ & $36.04 \pm 4.92$ \\
\hline spliced & Constrained & 1980-2006 & $567.37 \pm 117.31$ & $68.42 \pm 11.84$ & $1391.36 \pm 117.21$ & $34.62 \pm 4.83$ \\
\hline
\end{tabular}

The polar motion excitation tide model was determined by fitting residual observations spanning 2 January 1980 to 31 March 2002 when the c20010701 OGCM was used to remove non-tidal oceanic effects from the observations; by fitting residual observations spanning 2 January 1993 to 10 September 2006 when the kf066a2 OGCM was used; and by fitting observations spanning 2 January 1980 to 8 September 2006 when the c20010701/kf066a2 spliced OGCM was used. The uncertainties given for the estimated parameters are the $1 \sigma$ formal errors from the weighted least-squares fit. No uncertainties are given for the $\mathrm{mtm}$ and $m f$ tidal coefficients that were determined from the constrained solutions because they are derived from the respective solutions for the $M t m$ and $M f$ coefficients. The amplitude and phase of the prograde and retrograde components of polar motion excitation are defined by Eq. (3). The units of the amplitudes are microarcseconds ( $\mu$ as). The preferred empirical polar motion excitation tide model is given in bold

Since the $M f$ and $m f$ tidal terms, as well as the $M t m$ and $m t m$ terms, differ in frequency by only $1 / 18.6 \mathrm{cpy}$, they should have the same relative response to the tide raising potential.
That is, they should have the same phase and an amplitude ratio that is the same as that of the tide raising potential at these frequencies. Table 2 shows that for the unconstrained 

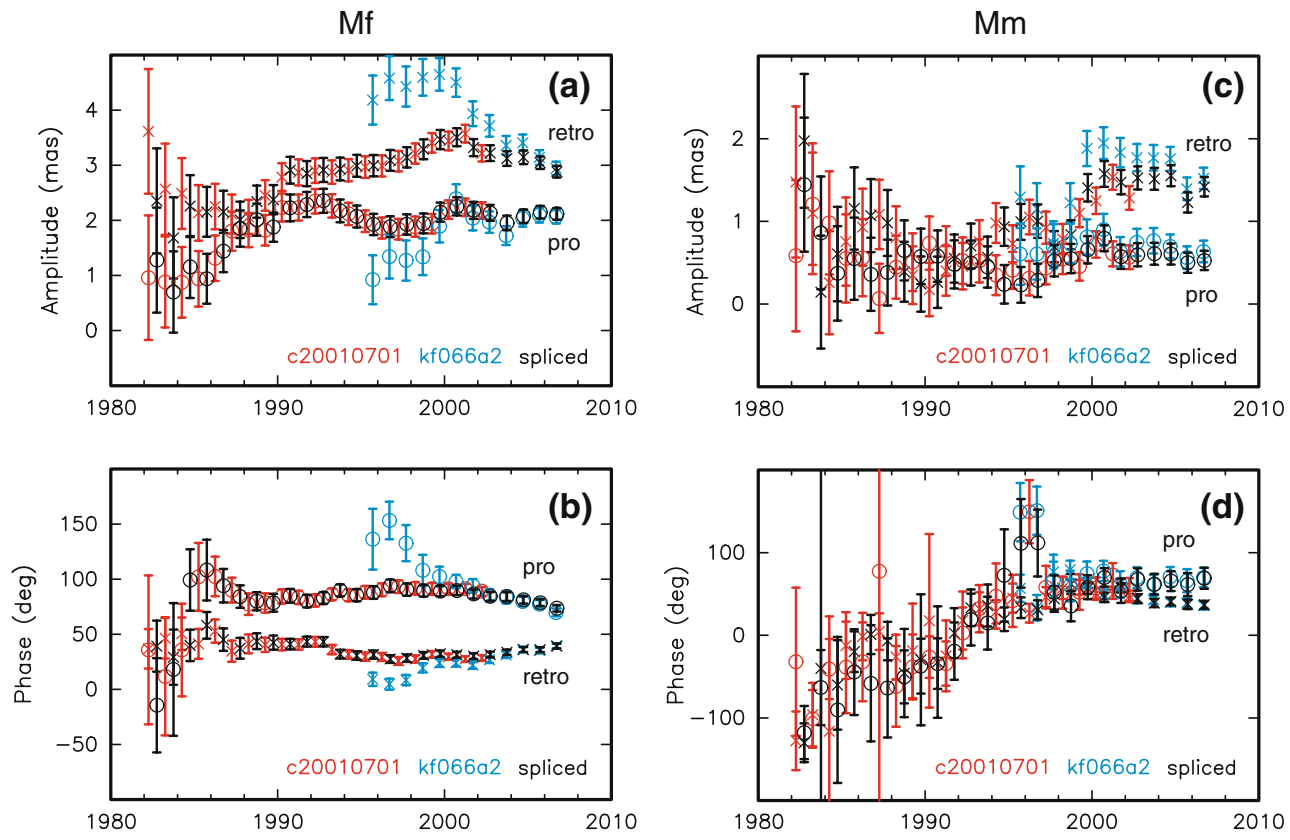

Fig. 2 Trial empirical polar motion excitation tide models determined from observations spanning increasing intervals of time. The amplitudes $\mathbf{a}, \mathbf{c}$ and phases $\mathbf{b}, \mathbf{d}$ of the prograde (pro; circles) and retrograde (retro; crosses) components of the recovered $M f \mathbf{a}, \mathbf{b}$ and $M m \mathbf{c}, \mathbf{d}$ tidal terms are shown as a function of the epoch of the last measurement that was used to determine the model. The error bars indicate the \pm 1 -sigma

formal uncertainties of the estimated amplitudes and phases. The units of the amplitudes are milliarcseconds (mas); the units of the phases are degrees (deg). Models determined by removing non-tidal oceanic effects using the c20010701 effective OAM values are shown in red, those using the kf066a2 values are shown in blue, and those using the spliced c20010701/kf066a2 values are shown in black

solutions the prograde $m f / M f$ amplitude ratios range from 0.475 to 0.566 depending on the particular OGCM that is used to remove the non-tidal oceanic effects, and the retrograde amplitude ratios range from 0.367 to 0.398 . While the retrograde amplitude ratios are reasonably close to the 0.4145 ratio of the amplitude of the tide raising potential at these frequencies, the prograde amplitude ratios are too large. The unconstrained solutions for the Mtm and $\mathrm{mtm}$ tidal terms have amplitude ratios that deviate even further from the 0.4146 ratio of the amplitudes of the tide raising potential at these frequencies, with the prograde $\mathrm{mtm} / \mathrm{Mtm}$ amplitude ratios ranging from 0.792 to 1.111 and the retrograde amplitude ratios being $0.262,0.769$, and 0.995 (depending on the particular OGCM that is used to remove the non-tidal oceanic effects). And rather than being the same, the unconstrained solutions for the (prograde, retrograde) phases of the Mtm and $m t m$ tidal terms differ by as much as $\left(89^{\circ}, 146^{\circ}\right)$, although the differences in phase between the $M f$ and $m f$ tidal terms are smaller, being at most $\left(7^{\circ}, 18^{\circ}\right)$.

In summary, except for the smaller Mtm tidal term, the solutions are relatively insensitive to the particular OGCM that is used to remove the non-tidal oceanic effects from the polar motion excitation observations. And applying constraints during the weighted least-squares fits for the empirical polar motion excitation tide model allows more accurate estimates to be obtained for the effects of those tidal

terms that are close to each other in frequency, namely, the $M f$ and $m f$ tidal terms and, separately, the Mtm and $m t m$ tidal terms.

\subsection{Convergence properties}

The convergence properties of the solutions were studied by fitting observations spanning increasing intervals of time. These convergence test solutions were all determined by constrained, weighted least-squares fits to polar motion excitation observations from which atmospheric and non-tidal oceanic effects were removed. Three different ocean models were used to remove the non-tidal oceanic effects in order to test the sensitivity of the convergence test solutions to the non-tidal ocean model. These were the same three models discussed in Sect. 3.2, namely, the c20010701 model, the kf066a2 model, and the spliced c20010701/kf066a2 model.

Figure 2 shows the recovered amplitudes and phases of the prograde and retrograde components of the $M f$ and $M m$ tidal terms of the convergence test solutions as a function of the epoch of the last measurement used to determine the solution. For example, a date of 2001.5 means that measurements through 2001.5 were used to determine that particular solution. As can be seen, the solution for $M f$ and, to a lesser extent, for $M m$ continues to slowly change as additional measurements after 2000 are included. Thus, the solutions for $M f$ 


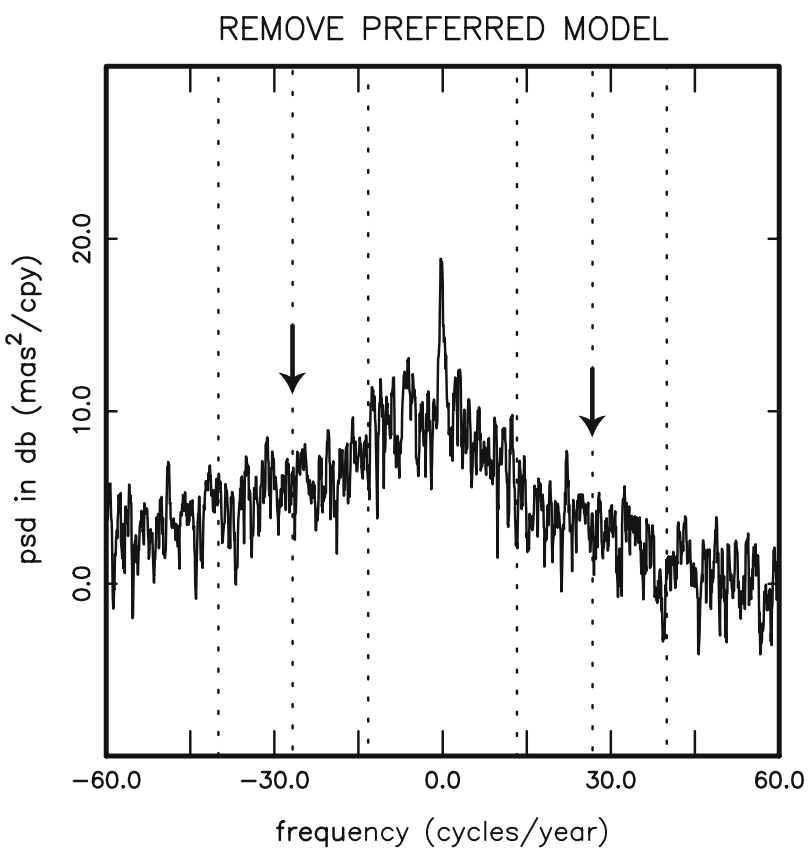

Fig. 3 Power spectral density (psd) estimates in decibels (db) computed by the multitaper method of residual polar motion excitation functions formed by removing atmospheric effects, non-tidal oceanic effects, and the preferred empirical tide model from observations spanning 2 January 1993 to 10 September 2006. Vertical dashed lines indicate the prograde and retrograde frequencies of the termensual $( \pm 40 \mathrm{cpy})$, fortnightly ( \pm 27 cpy) and monthly ( \pm 13 cpy) tidal terms. The prograde and retrograde fortnightly tidal terms are also indicated by arrows

and $\mathrm{Mm}$ may not have converged. Figure 2 also shows that the final solutions are relatively insensitive to the particular OGCM that is used to remove the non-tidal oceanic effects from the observations.

\subsection{Preferred model}

The preferred empirical model for the effects of the termensual (Mtm and $\mathrm{mtm}$ ), fortnightly ( $M f$ and $m f$ ), and monthly $(\mathrm{Mm})$ ocean tides on polar motion is that determined by a constrained, weighted least-squares fit for periodic terms at these tidal frequencies to polar motion excitation observations that span 2 January 1980 to 8 September 2006 and from which atmospheric effects were removed using effective AAM values computed from the products of the NCEP/ NCAR reanalysis model and non-tidal oceanic effects were removed using the spliced effective OAM values computed from the c20010701 and kf066a2 ocean models. The constraint applied during the fit is such that the periodic terms at the $M f$ and $m f$, and, separately, the $M t m$ and $m t m$, tidal frequencies are forced to have the same phase and an amplitude ratio that is the same as that of the tide raising potential at these frequencies. This model is preferred because it is based upon polar motion excitation observations spanning the longest possible time interval and because it should have more realistic estimates for the effect of the $M f$ and $m f$ tidal terms, and the Mtm and $\mathrm{mtm}$ tidal terms, because appropriate constraints were applied to these terms during the weighted least-squares fit. This preferred polar motion excitation tide model is given in bold in Table 2; it is reproduced in Table 1 as the entry labeled "This paper".

Figure 3 shows the spectrum of the result of removing the preferred empirical tide model from the residual polar motion excitation observations that span 2 January 1993 to 10 September 2006 and whose spectrum is shown in Fig. 1b. As can be seen, during this time interval the preferred model does an excellent job of removing the observed power at both the prograde and retrograde fortnightly tidal frequencies even though it was determined from observations that span a different time interval, namely, from observations that span 2 January 1980 to 8 September 2006.

\section{Discussion and summary}

An improved empirical model for the effect of long-period ocean tides on polar motion has been determined here by least-squares fitting to residual polar motion excitation observations periodic terms at the frequencies of the two principal termensual tidal constituents ( $\mathrm{Mtm}$ and $\mathrm{mtm})$, the two principal fortnightly tidal constituents ( $M f$ and $m f$ ), and the principal monthly tidal constituent $(\mathrm{Mm})$. This model is an improvement over previously available empirical polar motion tide models (Gross et al. 1996; Gross et al. 1997) because it is based on a fit to observations that span a greater interval of time, that has had non-tidal oceanic effects as well as atmospheric effects removed from the observations prior to the fit, and that has had constraints applied during the fit to force the $M f$ and $m f$ and, separately, the $\mathrm{Mtm}$ and $\mathrm{mtm}$ tidal terms to have the same phase and the same amplitude ratio as that of the tide raising potential at these frequencies. Tests have shown that, except for the smaller Mtm tidal term, the new model is relatively insensitive to the particular OGCM that is used to remove the non-tidal oceanic effects from the polar motion excitation observations. Tests have also shown that the new model is able to completely remove the observed power at the prograde and retrograde fortnightly tidal frequencies, not only the power observed during the same time interval that was used to determine the model (not shown) but also the power observed during shorter time intervals such as that used for generating the spectrum shown in Fig. 3 .

But even though this new empirical model is an improvement over previous models, it is still a model based upon fitting observations. The $M f$ and, to a lesser extent, the $M m$ tidal terms are still slowly changing as additional observations are included in the fit and they therefore appear to have not yet converged. Also, there is no guarantee that the 
model will fit future observations just because it fits past observations. A model for the effect of long-period ocean tides on polar motion that is determined from a dynamical ocean tide model, and that is therefore independent of polar motion observations, is clearly needed. There may now be a long enough span of Topex/Poseidon and Jason-1 altimetric sea surface height measurements that it may now be feasible to determine a long-period polar motion tide model from a dynamical ocean tide model that has been constrained by these measurements.

Acknowledgments I thank S. Dickman for his thoughtful review that led to many improvements to this manuscript. The work described in this paper was performed at the Jet Propulsion Laboratory, California Institute of Technology, under contract with the National Aeronautics and Space Administration. Support for this work was provided by the Earth Surface and Interior Focus Area of NASA's Science Mission Directorate. The supercomputers used in this investigation were provided by funding from the JPL Office of the Chief Information Officer.

Open Access This article is distributed under the terms of the Creative Commons Attribution Noncommercial License which permits any noncommercial use, distribution, and reproduction in any medium, provided the original author(s) and source are credited.

\section{References}

Brosche P (1982) Oceanic tides and the rotation of the Earth. In: Fricke WG, Teleki G (eds) Sun and planetary system, Reidel, pp 179-184

Cartwright DE, Edden AC (1973) Corrected tables of tidal harmonics. Geophys J R Astr Soc 33:253-264

Chao BF (1994) Zonal tidal signals in the Earth's polar motion. Eos Trans AGU 75(44): 158

Chao BF, Ray RD (1997) Oceanic tidal angular momentum and Earth's rotation variations. Prog Oceanogr 40:399-421

Chao BF, Ray RD, Gipson JM, Egbert GD, Ma C (1996) Diurnal/ semidiurnal polar motion excited by oceanic tidal angular momentum. J Geophys Res 101(B9):20151-20163

Desai SD, Wahr JM (1995) Empirical ocean tide models estimated from TOPEX/POSEIDON altimetry. J Geophys Res 100(C12): 25205-25228

Dickman SR (1993) Dynamic ocean-tide effects on Earth's rotation. Geophys J Int 112:448-470

Eubanks TM (1993) Variations in the orientation of the Earth. In: Smith DE, Turcotte DL (eds) Contributions of space geodesy to geodynamics: earth dynamics. American Geophysical Union Geodynamics Series, vol 24. Washington, DC, pp 1-54

Greatbatch RJ (1994) A note on the representation of steric sea level in models that conserve volume rather than mass. J Geophys Res 99:12767-12771

Gross RS (1993) The effect of ocean tides on the Earth's rotation as predicted by the results of an ocean tide model. Geophys Res Lett 20(4):293-296
Gross RS (2003) The GGFC Special Bureau for the Oceans: Past progress and future plans. In: Richter B, Schwegmann W, Dick WR (eds) Proceedings of the IERS workshop on combination research and global geophysical fluids. IERS technical note No 30. Bundesamts für Kartographie und Geodäsie, Frankfurt, Germany, pp 131-138

Gross RS (2007) Combinations of Earth orientation measurements: SPACE2006, COMB2006, and POLE2006. Jet Propulsion Laboratory Publ 07-5, Pasadena, Calif

Gross RS, Hamdan KH, Boggs DH (1996) Evidence for excitation of polar motion by fortnightly ocean tides. Geophys Res Lett 23(14):1809-1812

Gross RS, Chao BF, Desai S (1997) Effect of long-period ocean tides on the Earth's polar motion. Prog Oceanogr 40:385-397

Gross RS, Fukumori I, Menemenlis D (2003) Atmospheric and oceanic excitation of the Earth's wobbles during 1980-2000. J Geophys Res 108(B8):2370. doi:10.1029/2002JB002143

Kalnay E, Kanamitsu M, Kistler R, Collins W, Deaven D, Gandin L, Iredell M, Saha S, White G, Woollen J, Zhu Y, Chelliah M, Ebisuzaki W, Higgins W, Janowiak J, Mo KC, Ropelewski C, Wang J, Leetmaa A, Reynolds R, Jenne R, Joseph D (1996) The NCEP/NCAR 40-year reanalysis project. Bull Am Met Soc 77:437-471

Marshall J, Hill C, Perelman L, Adcroft A (1997a) Hydrostatic, quasihydrostatic, and nonhydrostatic ocean modeling. J Geophys Res 102:5733-5752

Marshall J, Adcroft A, Hill C, Perelman L, Heisey C (1997b) A finitevolume, incompressible, Navier Stokes model for studies of the ocean on parallel computers. J Geophys Res 102:5753-5766

Salstein DA (2003) The GGFC Special Bureau for the Atmosphere of the International Earth Rotation and Reference Systems Service. In: Richter B, Schwegmann W, Dick WR (eds) Proceedings of the IERS workshop on combination research and global geophysical fluids. IERS technical note No 30. Bundesamts für Kartographie und Geodäsie, Frankfurt, Germany, pp 121-124

Seiler U (1991) Periodic changes of the angular momentum budget due to the tides of the world ocean. J Geophys Res 96(B6): 10287-10300

Simon JL, Bretagnon P, Chapront J, Chapront-Touzé M, Francou G, Laskar J (1994) Numerical expressions for precession formulae and mean elements for the Moon and the planets. Astron Astrophys 282:663-683

Stammer D, Wunsch C, Fukumori I, Marshall J (2002) State estimation improves prospects for ocean research. Eos Trans AGU 83(27):289-295

Weis P (2006) Ocean tides and the earth's rotation—results of a highresolving ocean model forced by the lunisolar potential. Ph.D. Thesis, Universität Hamburg

Wunsch C, Stammer D (1997) Atmospheric loading and the oceanic "inverted barometer" effect. Rev Geophys 35:79-107

Zhou YH, Salstein DA, Chen JL (2006) Revised atmospheric excitation function series related to Earth variable rotation under consideration of surface topography. J Geophys Res 111:D12108. doi:10. 1029/2005JD006608 